\title{
Research on model component method based on OpenMI technology
}

\author{
Haiyan $\mathrm{Liu}^{1}$, Xiaomin $\mathrm{Liu}^{2,}$, and Zhenhua $\mathrm{Xu}^{1}$ \\ ${ }^{1}$ School of Computer \& Communication Engineering, Beijing Information Technology College, \\ Beijng, China \\ ${ }^{2}$ Water Conservancy and Civil Engineering College of Inner Mongolia Agricultural University, Inner \\ Mongolia, China
}

\begin{abstract}
Open Model Interface (OpenMI) technology can construct standardized components with unified timing interface and standardized data interaction. This provides a standardized method for integrating multiple models with different interfaces and functions. This paper briefly introduces the operation principle of OpenMI technology and the process of model standardization, and describes the seven key steps of model transplantation by taking the one-dimensional hydraulic model of the Yellow River as an example.
\end{abstract}

\section{Introduction}

Multi model integrated simulation technology of water conservancy is to apply the interface technology of computer software to the model interface, focusing on the standardization of the interface between model in order to use the same interface format for any model and increase the convenience and scalability of model combination. The source code of model of traditional water conservancy industry is written in FORTRAN and C language, which is compiled and run on a single machine. It is not conducive to the usage efficiency of the model [1]. The European Commission on water framework has developed the standard of open model interface (OpenMI), which is a standard and rule for model integration. It can transplant and transform the models based on time series.

Many scholars use the standard interface of OpenMI to realize the coupling integration of models, for example, the Danish Hydraulic Institute developed the MIKE system software-MIKE SHE[2], Zhou Huaye put forward the distributed data interactive mechanism in the process of the model integrated model on the basis of the description and analysis for the timing driving integrated model application [3], Shi feiran developed model simulation platform based on cloud computing by using OpenMI technology standard [4], Lei Sihua etc. developed soil moisture prediction model by using OpenMI technology [5].

In this paper, we study the model componentization based on the standard interface of OpenMI and construct a standardized component with unified timing interface and standardized data interaction. This paper first introduces the operation principle of OpenMI

\footnotetext{
${ }^{*}$ Corresponding author: lhy_lj@sina.com
} 
technology, and then describes the standardization process of using OpenMI technology. Taking the one-dimensional hydraulic model of the Yellow River as an example, it introduces the key steps of transplanting and transforming the existing model.

In this paper, we study the model componentization based on the standard interface of OpenMI and construct a standardized component with unified timing interface and standardized data interaction. This paper first introduces the operation principle of OpenMI technology, and then describes the standardization process of using OpenMI technology. Taking the one-dimensional hydraulic model of the Yellow River as an example, it introduces the key steps of transplanting and transforming the existing model.

\section{Introduction of OpenMI model}

The schematic diagram of OpenMI model is shown in Figure 1 [6-8].

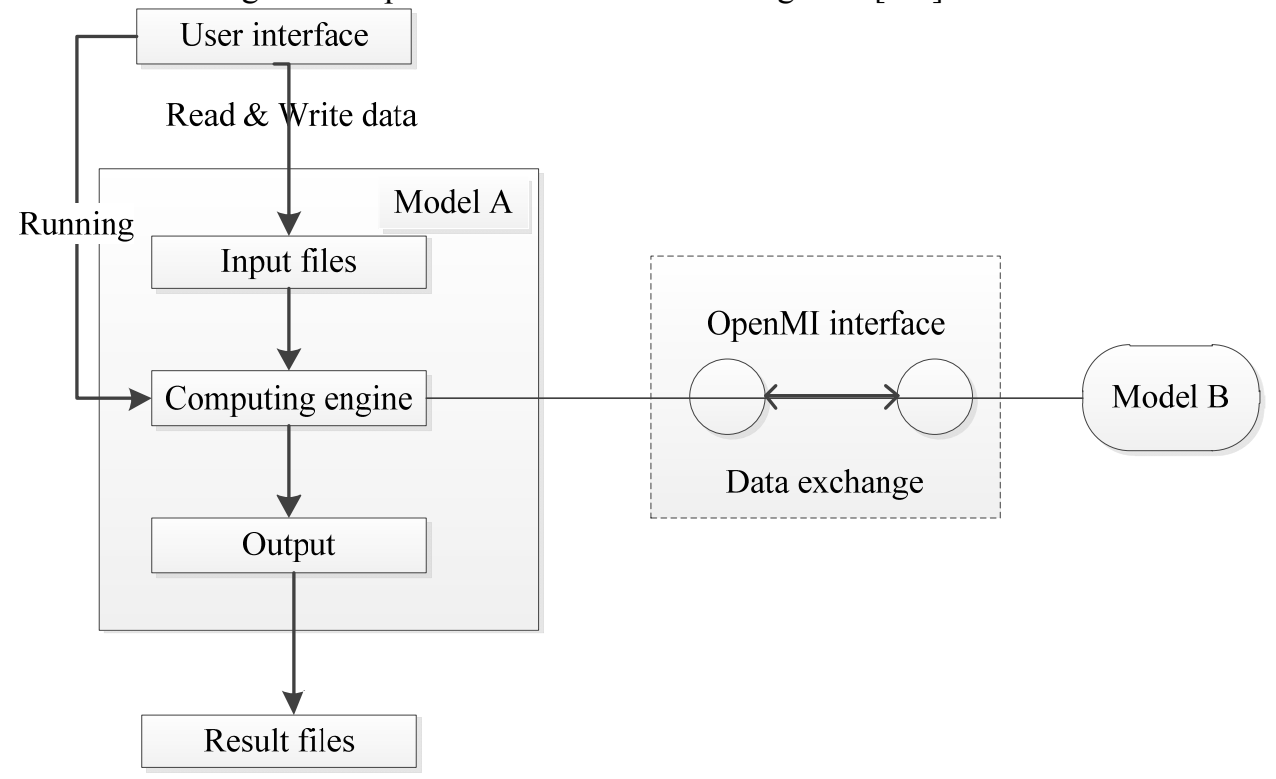

Fig. 1. Schematic diagram of OpenMI model

Both model A and model B are rewritten standardized components according to the requirements of OpenMI. Assuming that the input data of model B is the output data of model a, when the two models are coupled for simulation calculation, OpenMI will automatically complete the data transfer between models through the request response mechanism. The main functions of this mechanism include GetValues(time: Itime, linkID: string) and SetValues (time: Itime, linkID: string). The function GetValues allows data exchange between two connecting components. When the data request module needs to input data, call the GetValues function of the response module to issue instructions. The function SetValues is used to assign variables to the corresponding values obtained by the request data module [5].

\section{Model standardization process}

The standardization process of OpenMI is shown in the figure 2 [1]. 


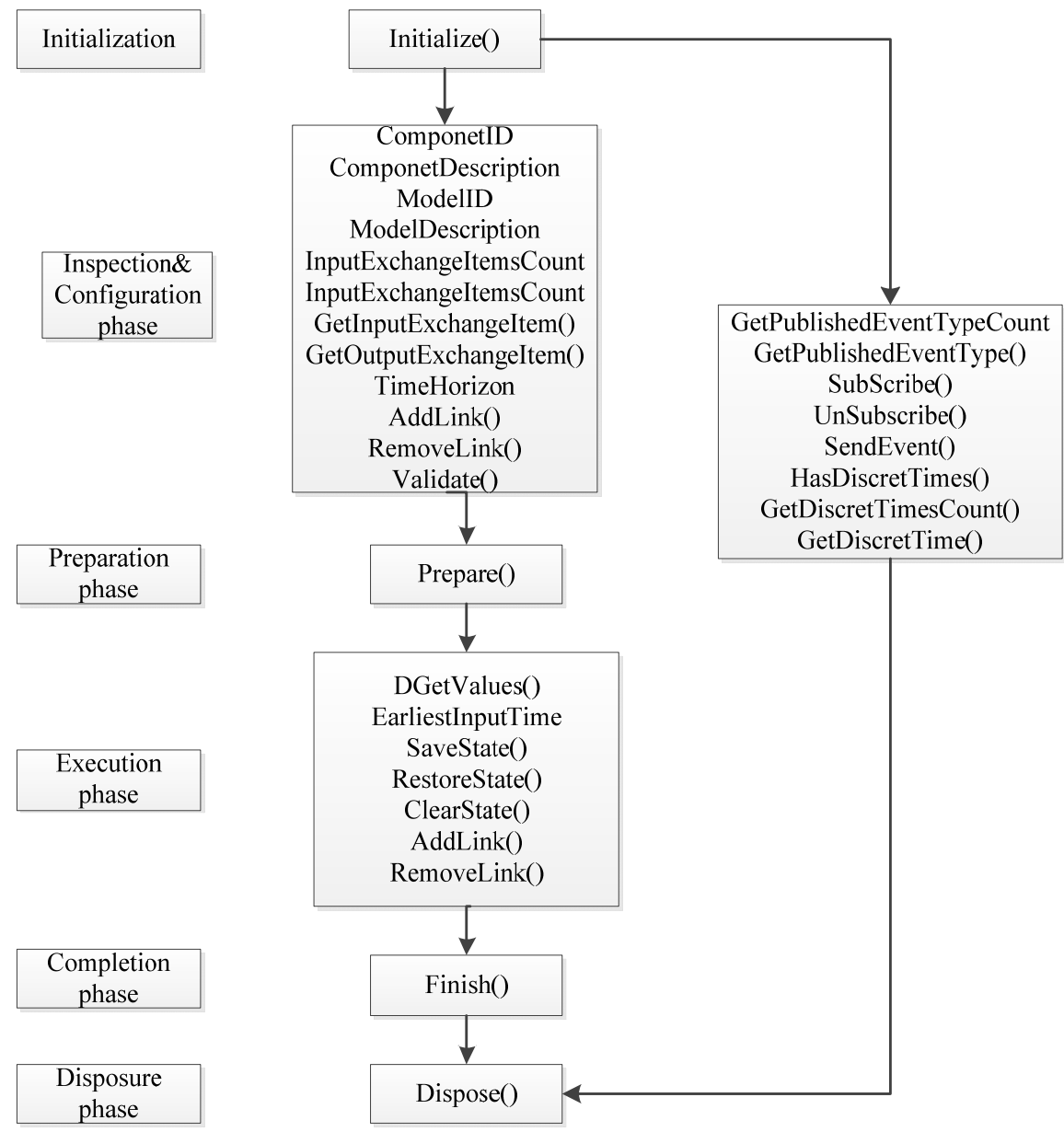

Fig. 2. Schematic diagram of OpenMI model

In the Initialization phase, LinkableComponet calls input data by function initialize(); in the inspection and configuration phase, it checks the validity of data operation and interaction items, creates connection module class, data interface class, element enumeration class object and defines data operation function, and uses validate() method to verify the status of components and their connection; in the preparation phase, the method of this phase is prepare (), which needs to establish the database and network connection, open the output file and organize the cache module; in the execution phase, the request response mechanism is used to complete the data transfer between the models; in the completion phase, Finish () is used to close the file and clear the network cache; in the disposure phase, the application is closed and the memory is released [10]. The inputexchangeitem is the interface of input data item and the outputexchangeitem is the interface of output data item.

\section{Model transplantation}

If we want to standardize the model of OpenMI, we need to transform and transplant the model. Taking the one-dimensional hydraulic model of the Yellow River as an example, this paper introduces the process of using OpenMI to transform and transplant the model, as 
shown in Figure 3. Firstly, the data processing process of the simulation model to be integrated is analyzed, and the running structure of the model is analyzed, and then the model is transplanted. The migration process is mainly divided into seven steps [11]: (1) change the engine core and compile the EXE file into DLL file; (2) create. Net components to ensure that the OpenMI environment has been installed successfully, load .Net environment, create packaging class components and corresponding test class components; (3) access the engine kernel function, execute MyEngineDotNetAccess class; this class is implemented through Initialize, PerformTimeStep, Fisnish, Dispose; (4) execute MyEngineWrapper class, change the call method to $\mathrm{C}$ \# form, change the error information to. Net processing, and get the error information through GetMessage method; (5) define MyEngineWrapper to implement the definition of ILinkableEngine class interface; (6) execute MyModeILinkableComponet class; (7) define other interface functions to complete the migration of models.

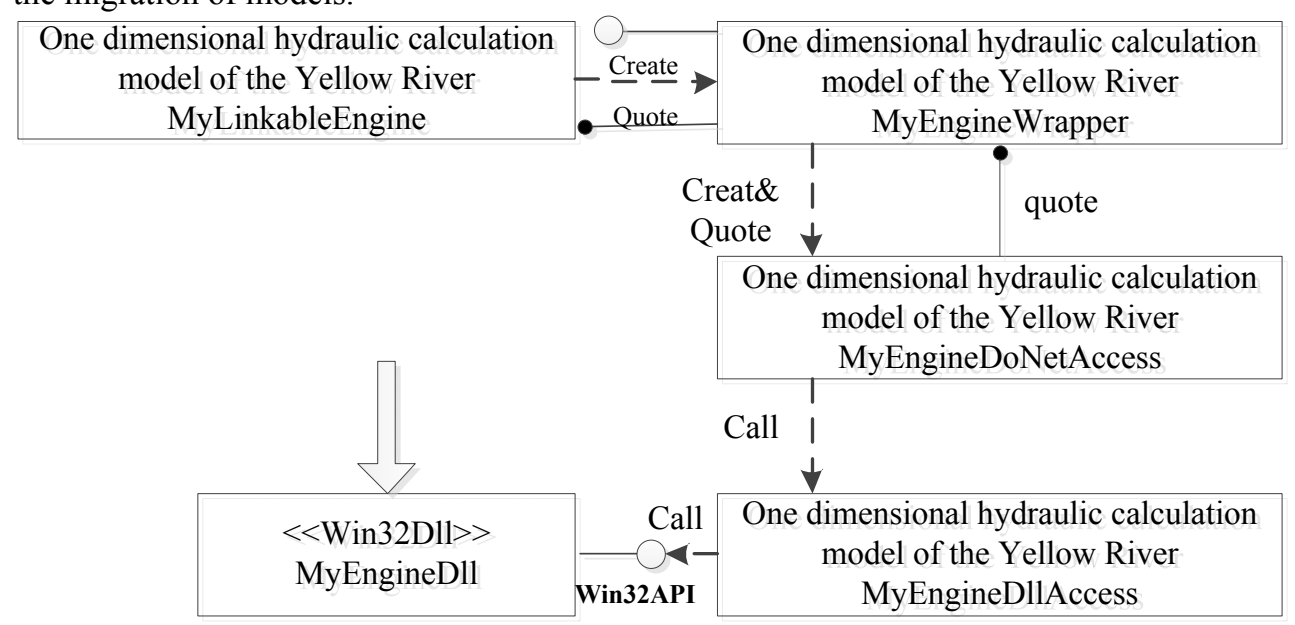

Fig. 3. The Process of model transplantation by using OpenMI

\section{Conclusion}

The model has a unified data interface and data interaction mode by using OpenMI standard to transform the model. In a certain integration or composition environment, the model that meets the requirements can freely connect and interact with data. It will improve the use efficiency of existing models and avoid the repetitive development. At the same time, through the integration and combination of existing models, the composite model can obtain more complex function that the single model does not have It has important practical significance and application prospects.

This research work was supported by R\&D Program of Beijing Municipal Education Commission (No.KM201810857001), The Major Science and Technology Projects of Inner Mongolia Autonomous Region (2020ZD0009), the National Natural Science Foundation of China (No.51969021)

\section{References}

1. XIA Runliang, FENG Xingkai, HE Mingmin, WU Hutong. Research on Cloud Service Platform for Hydraulic Numerical Stimulation based on Model Standardization[J]. Water Resources Informatization, 2015, 6:1-7. 
2. DHI. MIKEII: A Modeling System for Rivers and Channels Reference Manual[R]. DHI,2005.

3. Zhou Huaye. Research and Implemention on the Distributed Integration Multi-Models Technology[J]. Nanjing University of Aeronautics and Astronautics The Graduate School College of Computer Science and Technology), 2011.

4. Shi Feiran. Research on the Interface of the Cloud-based System Generation Platform for Hydrological Simulation[D]. School of Computer Science and Technology, Tianjin University, 2015.

5. LEI Si-hua, CHEN Ming, WEI Jun-biao, LI Shi-hong. Soil moisture forecast model based on OpenMI technology[J]. Hydro-Science and Engineering, 2013,3:33-37.

6. Gregersen J B, Gijsbers P J A, Westen S J P. OpenMI: Open Modeling Interface[J]. Journal of Hydroinformatics, 2007, 9(3):175-191.

7. Voinov A, Cerco C. Model integration and the role of data[J]. Environmental Modelling \& Software, 2010, 25(8): 965-969.

8. Yan Tingxia, Xu Jinduo, Lin Chen, et al. Research progress of openMI technology in the integration of hydrological model[J]. South-to-North Water Transfers and Water Science \& Technology, 2016, 14(5):25-27.

9. YANG De-quan, LI Guang-chi, CHENG Chuang-chuang. OpenMI and its application to coupling models[J]. Hydro-Science and Engineering, 2013, 1:76-80.

10. Fan Ziwu, Zhang Ming, Ma Zhenkun. Open model public interface OpenMI and its application[J]. Advances in hydraulics and hydraulic informatics, 2009, 1:676-680.

11. LEI Si-hua. Universal model interface for timing computation-OpenMI development technology and application[M]. Beijing. China Water Conservancy and Hydropower Press. 